\title{
Erratum to: Effect of Silica-Based Nanomaterials and Their Derivate with PEGylation on Cementoblasts
}

\author{
Xingfu Bao, Xiaoxi Wei, Yuzhuo Wang, Huan Jiang, Dongsheng Yu, and Min Hu \\ Department of Orthodontics, School of Stomatology, Jilin University, 1500 Qinghua Road, Changchun 130021, China
}

Erratum to: Annals of Biomedical Engineering

(2014) 42(8):1781-1789

DOI 0.1007/s10439-014-1012-X

This erratum is to correct for the wrong presentation of Fig. 5(c) and acknowledgments. Figure 5c was left without changing when preparing for Fig. 5 by using a template. So the image of apoptosis analysis under $\mathrm{PEG}-\mathrm{SiO}_{2}$ condition was not shown correctly, but the number of apoptosis ratio is correct. Figure 5 has been updated to show the right image by changing Fig. 5(c) only. The major findings and conclusions of this paper are not affected by these differences. The corrected version of Fig. 5 is presented here.

\section{ACKNOWLEDGMENTS}

The authors thank Professor Somerman and Ding Bai for providing cell line to them. This work was supported by National Natural Science Foundation of China, NSFC 81170999; Special Industrial Research supported by Development and Reform Commission of Jilin Province (2013C025-2); Foundation from Department of Health of Jilin Province, China, 2011Z091; and Specialized Research Fund for Doctoral Program of Higher Education, China, SRFDP 20110061110072.

Address correspondence to $\mathrm{Min} \mathrm{Hu}$, Department of Orthodontics, School of Stomatology, Jilin University, 1500 Qinghua Road, Changchun 130021, China. Electronic mail: humin@jlu.edu.cn

The online version of the original article can be found under doi: 10.1007/s10439-014-1012-x. 

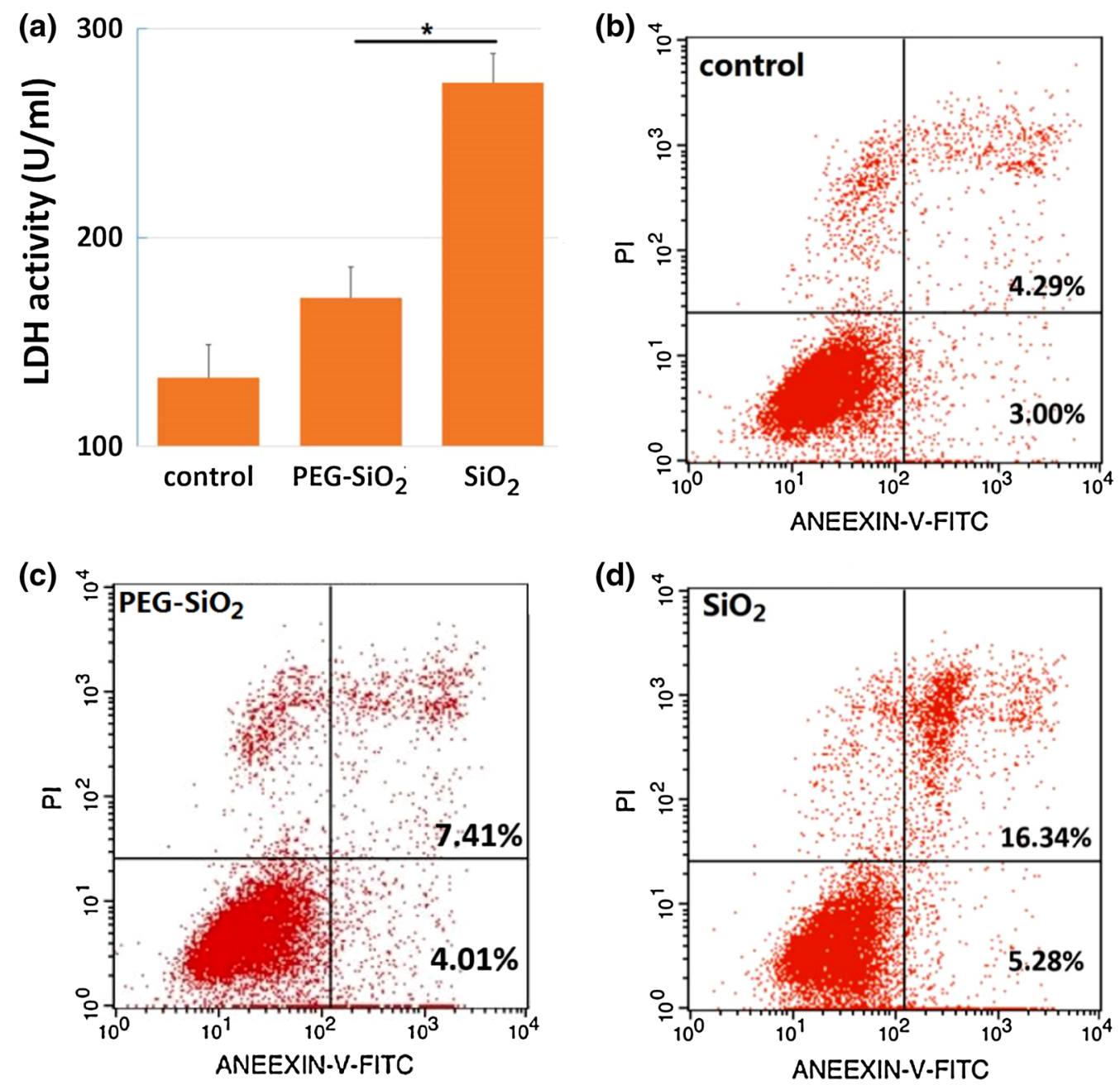

FIGURE 5. Quantitative analysis of LDH under different incubation conditions (a). Apoptosis analysis of cementoblasts in different treatment groups $(b-d)$. 